\title{
Profiling Turkish Honeys to Determine Authenticity Using Physical and Chemical Characteristics
}

\author{
Hamide Z. Senyuva,,${ }^{* \dagger}$ John Gilbert,${ }^{\star}$ Sibel Silici, ${ }^{\S}$ Adrian Charlton, ${ }^{\dagger}$ Cansu Dal,, \\ Neslihan Gürel, ${ }^{\dagger}$ and Dilek Cimen ${ }^{\perp}$ \\ ${ }^{\dagger}$ Ankara Test and Analysis Laboratory, Scientific and Technological Research Council of Turkey, Ankara 06330,

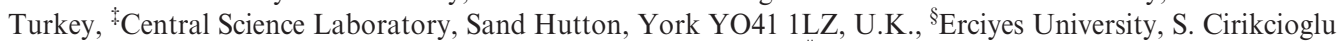 \\ Vocational Collage, Department of Animal Science, Kayseri, Turkey, "Bilkent University, Department of Chemistry, \\ Main Campus, 06800 Ankara, Turkey, and ${ }^{\perp}$ Gazi University, Department of Chemistry, Besevler, 06110 Ankara,
} Turkey

\begin{abstract}
Seventy authentic honey samples of 9 different floral types (rhododendron, chestnut, honeydew, Anzer (thymus spp.), eucalyptus, gossypium, citrus, sunflower, and multifloral) from 15 different geographical regions of Turkey were analyzed for their chemical composition and for indicators of botanical and geographical origin. The profiles of free amino acids, oligosaccharides, and volatile components together with water activity were determined to characterize chemical composition. The microscopic analysis of honey sediment (mellissopalynology) was carried out to identify and count the pollen to provide qualitative indicators to confirm botanical origin. Statistical analysis was undertaken using a bespoke toolbox for Matlab called Metabolab. Discriminant analysis was undertaken using partial least-squares (PLS) regression followed by linear discriminant analysis (LDA). Four data models were constructed and validated. Model 1 used 51 variables to predict the floral origin of the honey samples. This model was also used to identify the top 5 variable important of projection (VIP) scores, selecting those variables that most significantly affected the PLS-LDA calculation. These data related to the phthalic acid, 2-methylheptanoic acid, raffinose, maltose, and sucrose. Data from these compounds were remodeled using PLS-LDA. Model 2 used only the volatiles data, model 3 the sugars data, and model 4 the amino acids data. The combined data set allowed the floral origin of Turkish honey to be accurately predicted and thus provides a useful tool for authentication purposes. However, using variable selection techniques a smaller subset of analytes have been identified that have the capability of classifying Turkish honey according to floral type with a similar level of accuracy.
\end{abstract}

KEYWORDS: Turkish honey; amino acids; disaccharides; floral types; chemometrics

\section{INTRODUCTION}

The analytical characterization of honey as a means of detecting adulteration or of verifying geographical or botanical origin has been a scientific preoccupation for at least the past 30 years. Notwithstanding the considerable efforts that have gone into assessing a variety of parameters that characterize honey, there is still no definitive approach that is sufficiently rigorous to withstand legal challenge. The classical method of pollen analysis still has a part to play in distinguishing floral types of honey $(1)$ and to an extent in determining geographical origin, although pollen type is not an unequivocal indicator of country of origin, and fraudulent addition of pollen is not unknown.

Aroma is an important quality factor in honey reflecting nectar composition and floral origin. Honey of unifloral origin usually commands a higher price as do certain specific floral types, and thus, volatile analysis has been developed to distinguish on the

*Corresponding author. TUBITAK-ATAL, no. 67, Besevler, 06530 Ankara, Turkey. Tel: +90 312 2124620/ext.14. Fax: +90 312 2124620. E-mail: hamide.senyuva@tubitak.gov.tr. basis of floral type. From an analytical perspective, the high number of volatile components in honey provides a unique aroma profile representing a fingerprint of the product, which lends itself to multivariate data handling. Nevertheless, some single compounds or groups of compounds have been reported as indicative of floral type, e.g., phenylacetaldehyde, nonanoic acid, acetophenone, decanoic acid, benzaldehyde, phenylacetonitrile, isophorone, and nonanal being reported as characteristic markers of thyme honey (2); nonanol, nonanal, nonanoic acid, and acetoin as being characteristic of eucalyptus honey (3); acetophenone, 1-phenylethanol, and 2-acetophenone being characteristic of chestnut honey (4); and isophorone as a possible marker for heather honey (5). For aroma analysis, the approach of using solid phase microextraction (SPME) has been a major advance $(2$, $6)$, providing a fast and simple approach avoiding the risks of artifact formation through heating, and when combined with GC/MS providing unequivocal characterization of key volatiles.

Free amino acids provide a direct link to pollen content and thus have also been used to relate to botanical and geographical origin of honey. Proline is by far the most dominant amino acid, 
but it has been either the ratio of minor individual amino acids or more complex profiles that have been used for fingerprinting honey. The amino acids arginine, tryptophan, and cystine have been shown to be characteristic of some floral types, but there is considerable variability in patterns as even unifloral honey will have a complexity depending on differences in pollen availability. This may also be reflected in year-to-year variations, which need to be assessed in any proposed methodology built on authentic data sets. Most studies have focused on looking for differences in floral origin of honeys from a single geographical region such as Spain (7) or have looked directly at the bee pollen itself and corresponding honey to identify marker compounds (8). With this approach, free $\alpha$-aminobutyric acid was extensively found in bee pollen, and $\gamma$-aminoadipic acid and homoserine were detected for the first time in monovarietal honey samples from ilex, oak, heather, and chestnut (8). Earlier work used profiling of 17 free amino acids to discriminate honey based on geographical origin (9). Good discrimination was shown between samples from Australia, Argentina, and Canada, but U.K. honeys were not a tight group and fell between those samples from Argentina and Canada. Overall, it seems that amino acids give some indications of botanical and geographical sources but by themselves are not sufficient to provide unequivocal discrimination.

The other main parameter, which has been frequently explored for discrimination, has been oligiosaccharide composition of honey. Honey principally comprises glucose and fructose but also contains a complexity of minor oligosaccharides in variable amounts. It has for example been suggested that the contents of maltose, nigerose, turanose, and maltotriose could be used to differentiate honey samples from Brazil (10). A total of 40 individual oligosaccharides were measured in 91 samples of authentic U.K. honey (11), and using canonical discriminant analysis, it was possible to distinguish heather, bramble, and rapeseed samples but not clover honey, which was misclassified. The authors did not report which oligosaccharides were the largest contributors to the discrimination.

A number of other parameters such as metals and minerals (12), flavanoids (13), organic acids (14), and phenolic compounds (15) have been measured and attempts made to use these to characterize honeys. Generally with complex sets of parameters such as volatiles, amino acids, polysaccharides, metals, and so forth, there has been no attempt to use multivariate statistical techniques other than for handling individual components within a single data set. The exception seems to be where simpler physicochemical parameters have been used such as total ash combined with acidity and viscosity (16); moisture combined with $\mathrm{pH}$, electrical conductivity, hydroxymethylfurfural (HMF), and color (17); and water content combined with electrical conductivity, $\mathrm{pH}$, free acidity, invertase activity, HMF, total polyphenols, and free amino acids (18), although the latter were used to try to characterize a traditional honey-based product rather than discriminating between individual honeys.

In a recent review (19), it was concluded that aliphatic organic acids, amino acids, aroma compounds, aromatic carbonyl compounds, flavanoids, oligosaccharides, phenolic acids, and esters, proteins, and specific stable isotope ratios can in combination be indicative of botanical origin, while amino acids, aroma compounds, flavanoids, trace elements, oligosaccharides, protein, and specific stable isotope ratios can be used as indicators of geographical origin. In conclusion, it would seem that the application of sophisticated multivariate data-handling methods applied to a combination of different analytical parameters should be the best way forward to provide better discrimination (19), although to date, this approach has not been widely applied.
Geographical and climatic conditions in Turkey are particularly suitable as an environment for apiculture, where 4.5 million bee colonies, lead to an average production of $60-74,000$ tonnes of honey each year. This honey is particularly rich in diversity, and $85 \%$ of the world's floral types can be found in honey from Turkey. For example, honey from the Anatolia region of Turkey has been analyzed for pollen content (20), and even within this narrow geographical region on the Mediterranean coast, there were from 14 to 36 pollen types per sample of honey. Anzer honey is made from nectar collected from thymus spp. in the Artvin and Erzurum regions of Turkey and has been much studied in terms of its potential medicinal properties. Deli bali is a monofloral honey made from the spring flowers of the rhododendron (R. ponticum) that thrive on the humid Black Sea mountains. The nectar of the blooms are purported to contain andromedotoxin, a substance that can cause a range of physiological effects in humans such as tingling, numbness, and blurred vision to psychedelic effects. Although honey types are produced in Turkey, from a wide variety of botanical sources such as acacia, thyme, eucalyptus, orange blossom, heather, chestnut-tree, rhododendron, and honeydew, there has been only limited compositional analysis or attempts to classify by botanical or geographical origin.

In this article, for the first time we have characterized a wide range of different authenticated floral types of honey from Turkey using pollen analysis and water activity. Additionally, we have measured free amino acids, oligosaccharides, volatiles, and water activity in these samples and then have used number of different multivariate techniques to look for key indicators that can be used for discrimination of floral type, combining parameters from each of the different data sets.

\section{MATERIALS AND METHODS}

Chemicals. Alanine (Ala), arginine (Arg), asparagine (Asn), aspartic acid (Asp), cysteine (Cys), cystine (Cys-Cys), glutamic acid (Glu), glutamine (Gln), glycine (Gly), histidine (His), hydroxyproline (Hyp), leucine (Leu), isoleucine (Ile), lysine (Lys), methionine (Met), phenylalanine (Phe), proline (Pro), serine (Ser), threonine (Thr), tyrosine (Tyr), tryptophan (Trp), valine (Val), and benzophenone standards (99\%) were supplied by Aldrich (Milwaukee, USA). Raffinose, maltose, glucose, fructose, and saccharose were supplied by Dr. Ehrenstorfer $\mathrm{GmbH}$ (Augsburg, Germany). Formic acid (98\%) and acetic acid (glacial) were of analytical grade and obtained from Merck (Darmstadt, Germany). Ultrapure water was used throughout (Milli-Q system, Millipore, Bedford, MA, USA).

Honey Samples. A total of 70 samples $(200 \mathrm{~g})$ of each of nine different floral types of honey [rhododendron (20 samples), chestnut (6 samples), honeydew (6 samples), Anzer (8 samples), eucalyptus (2 samples), gossypium (2 samples), citrus (1 sample), sunflower (1 samples), and multifloral (24 samples)] were obtained from 15 different regions across Turkey, representing the important honey producing areas. The origin of the honey samples is shown on the map in Figure 1. Honeydew honeys were obtained in October 2005, and all other honeys were obtained in the 2006 honey season.

Extraction of Honey Sediment for Microscopic Analysis. The preparation of honey samples followed the standardized method (1) by which $10 \mathrm{~g}$ of homogenized honey was dissolved in $20 \mathrm{~mL}$ of distilled water and centrifuged for $10 \mathrm{~min}$ at $1,350 \mathrm{~g}$ (ca. 2,500 rpm) (21). The decanted sediment was washed with 5 to $10 \mathrm{~mL}$ of distilled water. After another centrifugation, the sediment was resuspended in $5 \mathrm{~mL}$ of $1: 1$ glycerine/distilled water and then centrifuged again, decanted, and mounted with glycerin jelly on microscope slides, which were sealed with paraffin. The pollen types present in the honey samples were identified, counted, and classified, according to their percentages, as dominant pollen (more than $45 \%$ of the total pollen grains counted), secondary pollen (from 16 to $45 \%$ ), important minor pollen (from 3 to $15 \%$ ), and minor pollen (less than $3 \%$ ). Counts were expressed as percentages after counting a minimum of 1000 pollen grains on four 


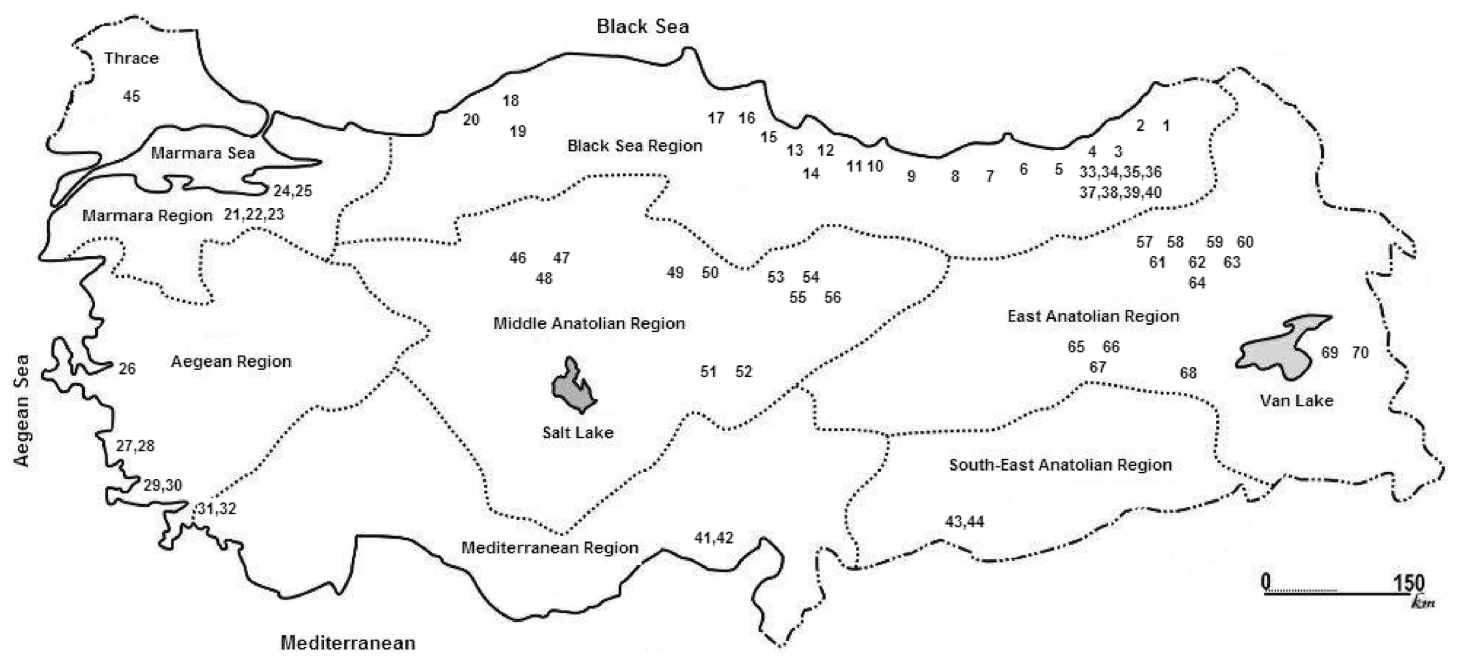

Figure 1. Map of Turkey showing the geographical distribution of honey samples. Rhododendron sample numbers, 1-20; chestnut sample numbers, 21-26; honeydew sample numbers, 27-32; Anzer sample numbers, 33-40; eucalyptus sample numbers, 41 and 42; gossypium sample numbers, 43 and 44 ; sunflower sample number, 45 ; and multifloral sample numbers, $46-70$.

slides from each sample. The examination of the pollen slides were carried out with an optical microscope at $\times 400$ and $\times 1000$ magnification in order to visually identify the pollen types. (20).

LC/MS Analysis of Free Amino Acids. Twenty-two free amino acids were determined directly in honey without sample cleanup and without derivatization using a fast amino acid method reported elsewhere (22). A homogenized sample of honey $(1 \mathrm{~g})$ was weighed into a $10 \mathrm{~mL}$ glass centrifuge tube with a cap, and $0.2 \mathrm{mM}$ acetic acid $(9.8 \mathrm{~mL})$ was added. After vortex mixing for $2 \mathrm{~min}$, it was centrifuged at $5000 \mathrm{rpm}$ for $10 \mathrm{~min}$ at $-5^{\circ} \mathrm{C}$. The clear supernatant was quantitatively transferred into a vial, avoiding the top oily layer if present, and was filtered through a $0.45 \mu \mathrm{m}$ nylon syringe filter. Samples were directly injected onto a narrow bore column (Zorbax, Bonus RP $2.1 \times 100 \mathrm{~mm} 3.5 \mu \mathrm{m}$ ) using an acidified mobile phase (0.01 mM HAc $+0.2 \%$ formic acid). LC/MS analysis employed an Agilent 1100 HPLC system (Waldbronn, Germany) consisting of a binary pump, an autosampler, and a temperature controlled column oven, coupled to an Agilent 1100 MS detector. Amino acids were determined by selected ion monitoring with LC/MS in positive atmospheric pressure chemical ionization (APCI) mode. Under these fast chromatography conditions, the retention times of all 22 amino acids were in the window from $0.9-7.5 \mathrm{~min}$, and the whole analysis was completed in $<25 \mathrm{~min}$.

SPME GC/MS Analysis of Honey Volatiles. The volatile profiles of honey samples were established using a solid phase micro extraction (SPME) method (3). Samples of honey $(6 \mathrm{~mL})$ dissolved in water ( $3 \mathrm{~g}$ honey $\mathrm{mL}^{-1}$ ) were dispensed into $20 \mathrm{~mL}$ headspace vials. Into each vial, $20 \mu \mathrm{L}$ of a solution of $10 \mu \mathrm{g} / \mathrm{mL}$ benzophenone in methanol was injected and the vial sealed with a PTFE septum. A carboxen polydimethylsiloxane (CAR/PDMS) fiber was suspended in the headspace and incubated at $60{ }^{\circ} \mathrm{C}$ for $10 \mathrm{~min}$ prior to subsequent headspace GC/MS analysis. GC/MS was carried out using an Agilent model $6890 \mathrm{GC}$ and $5973 \mathrm{MS}$ using an HP-5MS column $(30 \mathrm{~m} \times 250 \mathrm{~mm}$ $\times 0.25 \mu \mathrm{m}$ ) operated with a $1 \mathrm{~mL} / \mathrm{min}$ flow rate of helium. The column was held at $40{ }^{\circ} \mathrm{C}$ for $3 \mathrm{~min}$, then programmed at $3{ }^{\circ} \mathrm{C} / \mathrm{min}$ with a dynamic ramp to $160{ }^{\circ} \mathrm{C}$, followed by a further dynamic ramp at $10^{\circ} \mathrm{C} / \mathrm{min}$ to $200^{\circ} \mathrm{C}$ where it was held for $10 \mathrm{~min}$. Volatiles were initially identified by matching their electron ionization spectra obtained over the range $m / z$ 40-500 with NIST05, Wiley7, Flavor mass spectral database. Tentative identification was on the basis of good agreement with database spectra based on a criteria that a quality score from the database should be better than 80 out of 100 . Furthermore, the purity of all spectra were confirmed by reanalysis on a polar column, reconfirmation with database spectra, and running of standards typical of each class of compound. Peak areas were normalized to that of the benzophenone internal standard and were subsequently quantified by expressing the ratio of their total ion current response, assuming equivalent response factors. Absolute peak areas were used in the multivariate analysis.
HPLC Analysis of Raffinose, Sucrose, Maltose, Fructose and Glucose. A stock solution of oligiosaccharides $(15 \mathrm{mg} / \mathrm{mL})$ was prepared by dissolving $150 \mathrm{mg}$ of each in $10 \mathrm{~mL}$ of water. Working standards were prepared by diluting the stock solution to concentrations of $500-$ $5000 \mu \mathrm{g} / \mathrm{mL}$ with $50 \%$ acetonitrile. Stock solutions were kept at $4{ }^{\circ} \mathrm{C}$ for a week for daily use and kept at $-18^{\circ} \mathrm{C}$ for long term needs. Working standards were prepared daily before analysis. The homogenized honey sample $(1 \mathrm{~g})$ was weighed into a $10 \mathrm{~mL}$ glass centrifuge tube with a cap, and water $(10 \mathrm{~mL})$ was added. After it was mixed in a vortex mixer for $2 \mathrm{~min}$, it was centrifuged at $5000 \mathrm{rpm}$ for $10 \mathrm{~min}$ at $-5^{\circ} \mathrm{C}$. The clear supernatant was quantitatively transferred into a vial, avoiding the top oil layer if present. After filtering through a $0.45 \mu \mathrm{m}$ nylon syringe filter, LC analysis was carried out using an Agilent 1100 series liquid chromatograph equipped with a refractive index detector (RID), ALS autosampler, and thermostatted column module. The mobile phase was water with a $300 \mathrm{~mm} \times 7.8 \mathrm{~mm}$ i.d. Biorad Aminex HPX 87P LC column operated at $80^{\circ} \mathrm{C}$ at a flow rate of $0.6 \mathrm{~mL} / \mathrm{min}$. The injection volume was $20 \mu \mathrm{L}(11)$.

Determination of Water Activity. Water activity was determined at $25{ }^{\circ} \mathrm{C}$ using an electronic dew-point water activity meter, Rotronic $\mathrm{Ag}$ HYGROLAB (23). The equipment was calibrated with saturated salt solution in the $a_{\mathrm{w}}$ range of interest. For each determination, at least five replicates were made, and the results reported are the average values.

Chemometric Methods. Statistical analysis was undertaken using a bespoke toolbox for Matlab called Metabolab. All data were Fisher scaled. Exploratory data analysis, including principal components analysis (PCA) and analysis of variance (ANOVA), was undertaken. Twenty principal components scores were calculated representing $93.01 \%$ of the variance within the data. Discriminant analysis was undertaken using partial least-squares (PLS) regression followed by linear discriminant analysis (LDA). All models were cross-validated using the leave-one-out method. The maximum number of PLS scores were used such that the data was not overfitted. Overfitting was judged to have occurred when the cross-validation classification rate began to decrease. Typically, this was when greater than 4 or 5 scores were included in the models. Four data models were constructed and validated. Model 1 used all analytical data (51 variables) to predict the floral origin of the honeys. This model was also used to identify the top 5 variable important of projection (VIP) scores, selecting those variables that most significantly affected the PLSLDA calculation. These data were remodeled to assess the use of variable selected data for classification purposes. Model 2 used only the volatiles data, model 3 the sugars data, and model 4 the amino acids data.

\section{RESULTS AND DISCUSSION}

Although the honey samples that were obtained for this study were procured from authentic sources of known provenence, pollen analysis was undertaken as an additional confirmation of 
the correct assignment of floral type. The results of the pollen analysis confirmed that all of the rhododendron honey samples could be classified as monofloral with $>45 \%$ of rhododenron pollen being predominant. However, in one instance a honey from the area of Mersin, which was supplied as being monofloral eucalyptus honey, had been misclassified and in fact was a monofloral rhododednron honey. For the purposes of this study, this sample was subsequently treated as a rhododendron honey. This demonstrated the importance of using the pollen analysis for confirmation purposes. Chestnut honey contained $92 \%$ chestnut pollen, citrus contained $75 \%$ orange pollen, sunflower contained $66 \%$ sunflower pollen, and gossypium contained $52 \%$ pollen from cottonseed species. Anzer honey, although predominantly Thymus spp., did in fact also contain different pollen from other plants. Anzer honey contained only Thymus praecox from Thymus spp., although Trifolium spp. Lotus corniculatus, Castanea sativa, Cynoglossum glochidiatum, and Helianthemum nummularium pollen were also detected in these samples. Among the honey samples that were supplied as multifloral, a surprising number had high percentages of single pollen species, e.g., thyme honey (50\%), mustard (87\%), and Astragalus spp. (67-85\%). The results of the pollen analysis were not used directly but provided reassurance of the identity when other parameters were used for classification purposes.

We felt that it was important to have an indication of the quality of the samples of honey used in this study, and measurement of water activity was used as an indicator of viscosity, degree of crystallization, production distance from the sea, etc. It was also of interest to investigate whether water activity could of itself be used as a parameter together with any other indicators to discriminate honey floral types (see later). The physical state of honey in terms of whether it is in the crystallized form or the liquid state was found to influence water activity. Generally, crystallized samples showed higher water activity than liquified honeys. The difference in water activity between liquified and crystallized honeys was also found to be higher in flower honeys than in honeydew samples. Typically, rhododendron honey had a water activity in the range $0.458-0.618$, chestnut honey in the range $0.361-0.661$, honeydew honey in the range $0.44-0.61$, and Anzer honey in the range $0.38-0.557$. Thus, there was some overlap in the range of water activity for the main floral types of honey, although there were indications of the possibility of distinguishing rhododendron honey from Anzer honey with the latter having a distinctly lower range of activity. The results for Turkish honey are consistent with those reported elsewhere where the water activity of honey is generally found to be below 0.6.

Initial exploratory data analysis was undertaken for the complete data set of amino acids, volatiles, saccharides, and water activity, and 9 samples were removed from the data set due to missing data entries, providing a data matrix of 61 samples and 51 measurements. These data were analyzed by principal components analysis (PCA) to determine the presence of data clusters, and a plot of PC1 vs PC2 is shown in Figure 2. Twenty PC scores were calculated and visually inspected. Following this initial analysis, further data were removed due to the low data representation in group structures. Subsequent analysis therefore focused on those data from rhododendron, Anzer, chestnut, and multifloral honeys. Analysis of variance was used to calculate $P$ values for each measurement, which are shown in Table 1. This approach highlighted those analytes that had the potential to discriminate between honey types. While measurements with $p<$ 0.00001 were found, no individual analyte was able to clearly separate the four different floral types of honey.

All botanical sources were modeled simultaneously using PLSLDA. A success rate of $25 \%$ was deemed to be achieved by

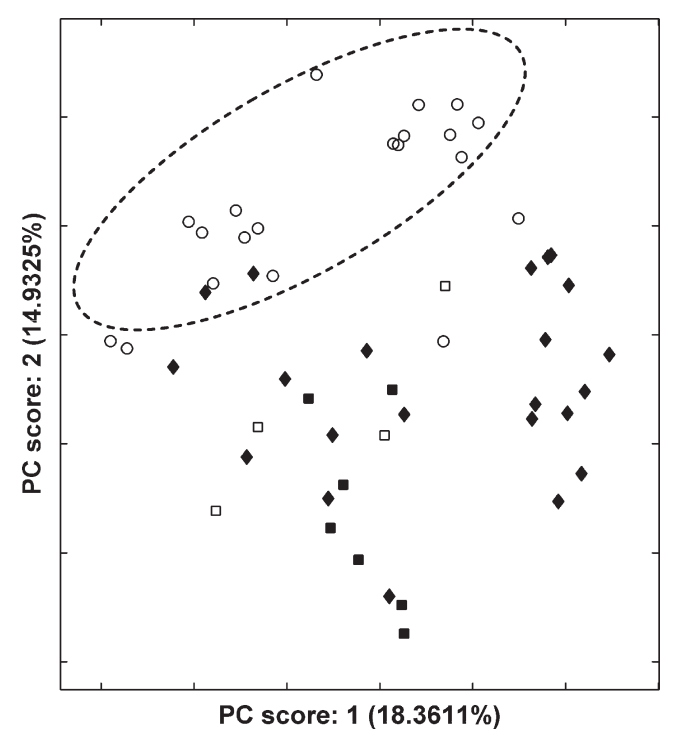

Figure 2. Exploratory data analysis using PCA highlights data clusters relating to the botanical origin of the honeys. PC1 and PC2 are plotted (with variance in parentheses), and these data clearly allow the separation of rhododendron honey from the other floral types. Rhododendron $(\bigcirc)$, multifloral $(\bullet)$, chestnut $(\square)$, and Anzer $(\square)$ honeys.

chance alone. Four data models were constructed and validated, and these are shown in Table 2. Model 1 used all of the 51 measurements to predict the floral origin of the honey samples. This model was also used to identify the top 5 variable important of projection (VIP) scores (shown in Table 1), selecting those variables that most significantly affected the PLS-LDA calculation. These data related to the analytes phthalic acid, 2-methylheptanoic acid, raffinose, maltose, and sucrose. Analysis of variance (ANOVA) on the data from these compounds showed that each $p<0.00001$ and that these were also the lowest $P$-values for any of the compounds assessed. Data from these compounds were therefore remodeled using PLS-LDA, and the classification rates shown in Table 2 were obtained. Model 2 used only the volatiles data, model 3 the sugars data, and model 4 the amino acids data. Classification and validation results for these models are also given in Table 2. Clearly, the combined data set allows the floral origin of Turkish honey to be accurately predicted and thus provides a useful tool for authentication purposes. However, using variable selection techniques a smaller subset of analytes has been identified that has the capability of classifying Turkish honey according to floral type with a similar level of accuracy.

It has long been recognized from the literature $(9-11)$ that amino acid profiles could be used as chemical markers for botanical and geographical origin of honey. Certain amino acids are derived from the bees themselves and are common to many honeys, while others originate from the pollen and are thus more characteristic of floral type. In this study, we have determined 22 free amino acids in all of the 70 honey samples. The mean levels of amino acids $(\mathrm{mg} / 100 \mathrm{~g})$ were in the following order of content Anzer $>$ chestnut $>$ eucalyptus $>$ rhododendron $>$ honeydew $>$ gossypium. Combinations of free amino acids were plotted to show the clustering of samples of common botanical origin. Good discrimination of Anzer, honeydew, and rhododendron honeys can be seen in Figure 3 based on the Fisher scaled phenylanine and tyrosine concetrations. It is evident that there were some deviations in the classification of the rhododendron honey, on the basis of these parameters, which were probably caused by the fact that the same unifloral rhododendron honey samples originated from different geographical regions in Turkey. The literature indicates that while amino acids have been successfully used for the 
Table 1. Analytes Ranked by Variable Importance of Projection (VIP) Score from a Partial Least Squares-Linear Discriminant Analysis (PLS-LDA) of Rhododendron, Anzer, Multifloral, and Chestnut Honey ${ }^{a}$

\begin{tabular}{|c|c|c|}
\hline analyte & $P$ value & VIP score \\
\hline phthalic acid & 0 & 2.0121 \\
\hline raffinose & 0 & 1.579 \\
\hline 2-methyl heptanoic acid & 0 & 1.5238 \\
\hline maltose & 0 & 1.4838 \\
\hline sucrose & 0 & 1.3688 \\
\hline tyrosine & 0.0002 & 1.3054 \\
\hline$p$-anisaldehyde & 0.0011 & 1.2621 \\
\hline valine & 0.0002 & 1.1948 \\
\hline linaloloxide & 0.0002 & 1.1736 \\
\hline n-eicosane & 0.0007 & 1.1014 \\
\hline phenyl acetaldehyde & 0.0005 & 1.0948 \\
\hline water activity & 0.0308 & 1.0632 \\
\hline leucine + isoleucine & 0.0055 & 1.0487 \\
\hline phenylalanine & 0.0021 & 1.0462 \\
\hline tryptophan & 0.009 & 1.0315 \\
\hline alanine & 0.0657 & 1.0302 \\
\hline aspartic acid & 0.0072 & 1.005 \\
\hline cysteine & 0.0253 & 0.9724 \\
\hline damascenone & 0.0023 & 0.9691 \\
\hline asparagine & 0.0181 & 0.9264 \\
\hline 4-carvomenthenol & 0.0178 & 0.9184 \\
\hline nonanol & 0.0241 & 0.9183 \\
\hline arginine & 0.0243 & 0.9161 \\
\hline p-cymene & 0.1295 & 0.9149 \\
\hline glutamic acid & 0.3864 & 0.87 \\
\hline phenylethyl alcohol & 0.0857 & 0.8697 \\
\hline 2-aminoacetophenone & 0.0277 & 0.8643 \\
\hline nonanal & 0.0856 & 0.8582 \\
\hline lysine & 0.1846 & 0.8409 \\
\hline$\alpha-\alpha$-dimethyl phenyl acetate & 0.1748 & 0.8351 \\
\hline threonine & 0.0494 & 0.8134 \\
\hline cystine & 0.0817 & 0.7917 \\
\hline serine & 0.0377 & 0.7867 \\
\hline hydroxyproline & 0.1127 & 0.7802 \\
\hline glutamine & 0.4310 & 0.7356 \\
\hline methionine & 0.1895 & 0.7298 \\
\hline glucose & 0.0976 & 0.7241 \\
\hline lilac aldehyde & 0.1578 & 0.7152 \\
\hline proline & 0.1264 & 0.6769 \\
\hline histidine & 0.8641 & 0.6489 \\
\hline fructose & 0.3544 & 0.6087 \\
\hline glycine & 0.5421 & 0.5363 \\
\hline benzene dicarboxylic acid & 0.3375 & 0.5009 \\
\hline ethyl benzoate & 0.7150 & 0.3555 \\
\hline$n$-decane & 0.9353 & 0.1985 \\
\hline
\end{tabular}

${ }^{a}$ The analytes with the highest VIP score had the greatest influence on the classification of honey by floral origin. $P$ values from an analysis of variance for each of the analytes are also shown.

Table 2. Classification Results Obtained Using PLS-LDA for the Classification of Rhododendron, Anzer, Chestnut, and Multifloral Honeys

\begin{tabular}{lccc}
\hline \multicolumn{1}{c}{ data set } & $\begin{array}{c}\text { number of PLS } \\
\text { scores in model }\end{array}$ & $\begin{array}{c}\text { classification (\%) } \\
\text { construction }\end{array}$ & $\begin{array}{c}\text { classification (\%) } \\
\text { validation }\end{array}$ \\
\hline all data: model 1 & 4 & 96.23 & 83.02 \\
volatiles: model 2 & 3 & 90.57 & 81.13 \\
sugars: model 3 & 3 & 64.15 & 50.94 \\
amino acids: model 4 & 3 & 71.70 & 52.83 \\
top 5 compounds & 3 & 79.24 & 77.36
\end{tabular}

classification of honey with high statistical correlation, there are apparent contradictions in terms of which amino acids are significant between different geographical data sets. In our PLSDA analysis (Figure 2), some 10 amino acids contributed in the top 20 ranking of variables contributing to discrimination of

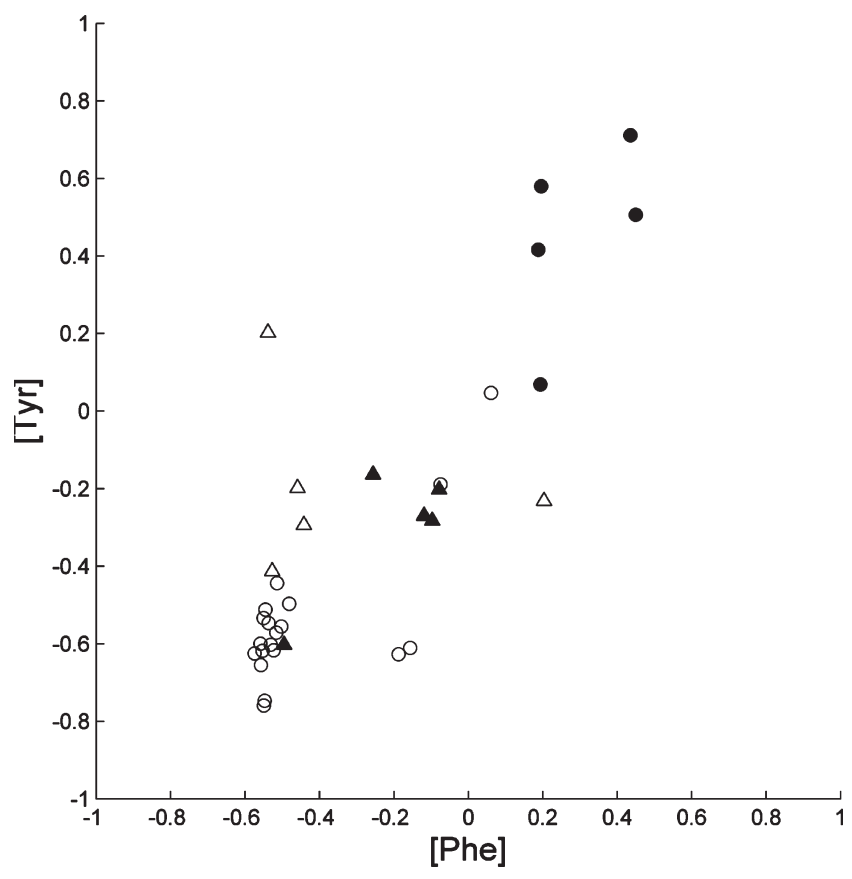

Figure 3. Discrimination of rhododendron $(O)$, chestnut $(\Delta)$, honeydew $(\boldsymbol{\Delta})$, and Anzer ( $\mathbf{O})$ Turkish honeys using the Fisher scaled tyrosine and phenylalanine content.

honeys (see Table 1). From the literature for Argentinian honey samples from three distinct geographical regions in the same country, different sets of between 5 and 7 amino acids were used to distinguish honeys from one individual region from another (24). Phenylalanine appeared as a critical amino acid in all three of the groups of amino acids, while tyrosine was only included in one group. Rosemary, eucalyptus, lavendar, thyme, and orange blossom honeys from Spain were compared for amino acid content (7), and while there was a complexity in the combinations of amino acids used in PCA, it was found that tyrosine could be used to distinguish lavendar honey from other botanical types. Phenylalanine and tyrosine used in combination as components in PCA provided $18.9 \%$ of the explained variance between these five floral honey types (7). Thus, the results for Turkish honey confirm the literature concerning the importance of phenylalanine and tyrosine as amino acids of significance in distinguishing honeys, although valine, leucine, and isoleucine also seem to make an important contribution to discrimination.

One study (25) revealed important amounts of tryptophan in sweet chestnut honey, but we have not been able to confirm that tryptophan is a significant amino acid in unifloral chestnut honey samples from Turkey. We found levels of tryptophan in chestnut honeys ranging from 0.32 to $0.75 \mathrm{mg} / 100 \mathrm{~g}$ (average $0.65 \mathrm{mg}$ / $100 \mathrm{~g}$ ) compared with average levels of 0.39 and $0.59 \mathrm{mg} / 100 \mathrm{~g}$ in rhododendron and honeydew honeys, respectively. Tryptophan ranked as 15th in variable importance of projection in Table 1 for the complete set of 51 variables.

The results for the SPME GC/MS headspace analysis of 61 of the 70 honey samples (unfortunately, for 9 samples there was insufficient honey available to carry out the analysis) are shown in Table 3 where both the principal and potential marker volatiles are indicated, on basis of a subjective sorting of the data indicating frequency of occurrence. In total, around 350 individual volatiles were identified as occurring in these samples, but while some were commonly found irrespective of botanical origin, there were no volatiles that were found to occur in all 61 samples. These results are not entirely consistent with other findings of commonly occurring volatiles (26) where of a total of 110 volatile 
Table 3. Principal and Potential Marker Volatiles Identified in Unifloral Turkish Honeys ${ }^{a}$

\begin{tabular}{|c|c|c|c|c|c|c|c|c|c|}
\hline \multirow[b]{2}{*}{ volatiles identified } & \multicolumn{9}{|c|}{ numbers of honey samples of different floral types containing key volatiles (total number of samples of each type) } \\
\hline & rho. (19) & chest. (4) & honey (2) & Anz. (7) & euca. (2) & multi. (23) & gossy. (2) & citrus (1) & sunfl. (1) \\
\hline$n$-decane & 14 & 3 & 2 & 5 & & 21 & & & \\
\hline lilac aldehyde & 10 & & & 5 & & 17 & & 1 & \\
\hline 2-aminoaceto phenone & 15 & & & 6 & & 7 & & & \\
\hline benzenedicarboxylic & 18 & & & 5 & & 23 & & & 1 \\
\hline nonanal & 17 & & 2 & 2 & 2 & 21 & 2 & & 1 \\
\hline isobutylphthalate & 15 & & & 3 & & 18 & & & \\
\hline damascenone & 14 & & & & & 18 & & & 1 \\
\hline $\begin{array}{l}\text { phenylacetaldehyde } \\
\text { hexadecanoic acid }\end{array}$ & & 3 & & 6 & & 17 & 2 & & 1 \\
\hline phthalic acid & & 4 & & & & 6 & & & \\
\hline$\alpha-\alpha$-dimethylphenyl acetate & & 4 & 2 & & & 10 & & & 1 \\
\hline p-anisaldehyde & & 3 & & & & 1 & & & 1 \\
\hline linaloloxide & & & & 6 & & 18 & & & \\
\hline$n$-eicosane & & & & 5 & & 19 & & & \\
\hline 4-carvomenthenol & & & & 3 & & 9 & & & 1 \\
\hline ethylphenyl acetate & & & & & 2 & & & & \\
\hline phenethyl alcohol & & & & & 2 & 14 & 2 & & \\
\hline nonanol & & & 2 & & & 12 & & & \\
\hline 2-methyl heptanoic acid & & & 1 & & & 20 & & & \\
\hline p-cymene & & & & & & 6 & & & 1 \\
\hline ethyl benzoate & & & & & & 1 & & & \\
\hline safranal & & & & & & & 1 & & \\
\hline cis-6-nonen-1-ol & & & & & & & & 1 & \\
\hline santene & & & & & & & & 1 & \\
\hline
\end{tabular}

${ }^{a}$ Rho, rhododendron; chest., chestnut; honey, honeydew; Anz., Anzer; euca., eucalyptus; multi., multifloral; gossy., gossypium; sunfl., sunflower.

compounds identified in honeys from nine floral sources, 17 volatiles were common to all 43 samples. This study used a sweep and trap method collecting volatiles on a Tenax trap prior to GC/MS identification, and while, for example, acetone, 2-butanone, methylbutanal, 2-propanol, ethanol, 2-pentanone, chloroform, and nonanal were common to all samples, other volatiles were shown to be characteristic of specific floral types (26). From the analysis of the volatiles from a wide range of Turkish honeys, we propose the presence of lilac aldehyde [2-(5-methyl-5-vinyltetrahydro-2-furanyl) propanal] and 2-aminoacetophenone as indicators of rhododendron honey. There is no previously published work on identified volatiles in rhododendron honey, although lilac aldehyde has been reported as a possible indicator for citrus honey from Greece (27) and has been found in honey from the Haze flower (28). Lilac aldehyde and lilac alcohol are themselves commonly occurring in flower scents and are believed to be involved as insect attractants (29). Solvent extraction of flowers and fruit of five Rhododendron species from Turkey showed the presence of some 200 compounds (30) including styrene and 2-ethylhexanol, which might be characteristic markers. However, styrene which seems surprising as a naturally occurring volatile has also been reported in chestnut honey (28) but could not be detected among the volatiles in this study. From our analysis of Turkish chestnut honey, $p$-anisaldehyde, which is a common flower volatile component with a woody smell ,is a proposed marker. Others (26) have proposed 2-methyldihydrofuranone or $\alpha$-methylbenzyl alcohol, or both 3-hexen-1-ol and dimethylstyrene as markers of chestnut honey. We could not detect 2-methyldihydrofuranone in any of the 70 Turkish honey samples, although $\alpha$-methylbenzyl alcohol was found in one of the six chestnut honeys and also in 8 rhododendron honeys, 1 Anzer honey, and 4 multifloral honeys. Thus $\alpha$-methylbenzyl alcohol did not appear to be particularly characteristic of chestnut honey from the results of our study. Neither 3-hexen-1-ol nor dimethylstyrene could be found in any of the 70 Turkish honey samples.
Anzer honey is unique to Turkey, and phenyl acetaldehyde, which is a minor component of many essential oils and fruits, is a proposed marker, although we also detected this compound in gossypium honey. Phenyl acetaldehyde has not been proposed elsewhere as a marker for other honeys but has previously been reported as present in heather, lavender, and lime honey (26). For eucalyptus honey, we propose nonanal as a marker compound, although we also detected nonanal in gossypium and honeydew honey, and it was reported elsewhere as occurring in all 43 honeys from 9 different floral sources (26). Others have proposed 1-octene or 2,3-pentanedione as markers for eucalyptus but neither of these compounds were found in Turkish eucalyptus honey nor indeed in any of the other samples of honey from other floral sources. Phenylethyl alcohol, which is found in a variety of essential oils, such as rose, carnation, hyacinth, and orange blossom, is proposed as a marker for gossypium honey, although it has been previously reported in eight different floral honeys but not detected in rosemary honey (26). No previous published studies appear to have included gossypium honey. For honeydew honey, we propose again that nonanal could be used as a marker but in combination with $\alpha, \alpha$-dimethyl phenyl acetate, the latter appears to be uniquely present in honey from this source. Methyl anthranilate has been proposed as a characteristic volatile for citrus honey (2). In this study, we did detect methyl anthranilate in the single sample of Turkish citrus honey, but we also found this marker in six of the multifloral honey samples, although the latter may have contained some citrus pollen.

The combinations of volatiles as shown in Table $\mathbf{3}$ can be used for the discrimination of honey from different floral sources. None of the volatiles is unique to any specific honey type. However, the groupings of volatiles shown are indicative of unifloral honeys. Thus, although not all chestnut honeys contained all four volatiles when three or four of any of phenyl acetaldehyde, phthalic acid, $\alpha, \alpha$-dimethylphenyl acetate, and $p$ anisaldehyde are present, these provide weight of evidence of a chestnut honey. Rhododendron honey, for example, contained 
Table 4. Ranges and Average Levels $(\mathrm{g} / 100 \mathrm{~g})$ of Principal Oligosaccharides in Honey Samples of Different Floral Type ${ }^{a}$

\begin{tabular}{|c|c|c|c|c|c|c|c|c|c|c|}
\hline \multirow[b]{2}{*}{ floral type } & \multicolumn{2}{|c|}{ glucose } & \multicolumn{2}{|c|}{ fructose } & \multicolumn{2}{|c|}{ maltose } & \multicolumn{2}{|c|}{ raffinose } & \multicolumn{2}{|c|}{ saccharose } \\
\hline & range & mean & range & mean & range & mean & range & mean & range & mean \\
\hline Anzer $^{b}$ & $21.1-35.7$ & 30.7 & $35.5-41.2$ & 37.7 & $<0.6$ & $<0.6$ & $0.3-0.8$ & 0.5 & $7.0-14.2$ & 11.6 \\
\hline rhododendron $^{c}$ & $16.4-37.8$ & 27.1 & $31.3-51.1$ & 39.6 & $2.6-7.7$ & 4.9 & $0.8-2.9$ & 1.8 & $10.3-24.9$ & 15.8 \\
\hline eucalyptus & $31.7-35.0$ & 33.4 & $37.6-39.6$ & 38.6 & $<0.6$ & $<0.6$ & $0.3-0.5$ & 0.4 & $6.6-7.2$ & 6.9 \\
\hline chestnut $^{d}$ & $26.0-44.8$ & 35.1 & $37.6-46.5$ & 42.7 & $<0.6$ & $<0.6$ & $0.1-0.4$ & 0.3 & $5.9-9.8$ & 7.3 \\
\hline honeydew & $20.2-21.8$ & 21.0 & $27.8-38.7$ & 33.3 & $6.3-6.8$ & 6.5 & $2.5-3.1$ & 2.8 & $14.9-15.6$ & 15.2 \\
\hline gossypium & $21.2-37.8$ & 29.5 & $32.3-43.3$ & 37.8 & $<0.6$ & $<0.6$ & $0.3-1.8$ & 1.0 & $7.1-16.3$ & 11.7 \\
\hline multifloral $^{e}$ & $21.6-33.2$ & 29.4 & $35.1-44.5$ & 38.3 & $3.3-5.1$ & 4.1 & $0.3-1.7$ & 0.7 & $7.2-16.6$ & 11.2 \\
\hline
\end{tabular}

${ }^{a}$ Single sample of citrus honey contained in $\mathrm{g} / 100 \mathrm{~g}$ glucose (33.5), fructose (39.3), maltose (<0.6), raffinose (0.5), and saccharose (9.2). Single sample of sunflower honey contained in $\mathrm{g} / 100 \mathrm{~g}$ glucose (31.4), fructose (35.5), maltose $(<0.6)$, raffinose $(0.4)$, and saccharose $(9.8) .{ }^{b}$ One sample of Anzer honey from Rize contained $4.41 \mathrm{~g} / 100 \mathrm{~g}$ of maltose. ${ }^{c}$ One sample of rhododendron honey from Ordu exceptionally contained $<0.6 \mathrm{~g} / 100 \mathrm{~g}$ of maltose. ${ }^{d}$ One sample of chestnut honey from Bursa exceptionally contained 1.8 $\mathrm{g} / 100 \mathrm{~g}$ of maltose. ${ }^{e}$ Only 5 samples of multifloral honey from the Black Sea region contained $3.3-5.1 \mathrm{~g} / 100 \mathrm{~g}$ of maltose.

none of these four volatiles but had a pattern of some seven characteristic volatiles, although again not all rhododendron honeys contained all seven volatiles. Thus, the use of volatiles is indicative and needs to be used in conjunction with other parameters. As expected, the multifloral honey samples contained nearly all of the volatiles listed in the characteristic groupings for monofloral honey. Table 3 does not provide enough detail to analyze the multifloral honey data or look for patterns in the multifloral honey samples. However, phthalic acid was ranked number 1 in variable importance (Table 1). Various phthalates have previously been reported among honey volatiles, but phthalic acid has not previously been suggested as a potential marker. The concentrations of phthalic acid for Turkish honeys have been plotted and are shown in Figure 4. Surprisingly, this shows an apparent separation of multifloral honeys from monofloral, which seems to indicate that despite containing a variety of pollens these samples had some common elements, which were not present in the monofloral honeys. Although these results do not help in distinguishing floral types, they might provide a useful tool for distinguishing multifloral honeys from those sold at a premium as being monofloral.

Despite the complexity of the patterns of volatiles, surprisingly few publications on volatiles have applied multivariate analysis to unravel this complexity and seek out those key marker compounds. Only in one study of thyme honey (2) from Greece by applying PCA to a data set of 63 volatiles, six components were extracted, explaining $85.4 \%$ of the total variance. The first component explained $46.2 \%$ of the variance and was positively correlated to phenyl acetaldehyde, nonanoic acid, acetophenone, decanoic acid, benzaldehyde, phenyl acetonitrile, isophorone, and nonanal (2). Of these eight volatiles, in our study only nonanal and phenylacetaldehyde were common volatiles in Turkish honey (see Table 3), and phenylacetaldehyde ranked as 11 th in importance as a variable contributing to discrimination (Table 1).

Although the analysis of sugars in honey has to a large extent focused on honey adulteration, oligosaccaride profiles are also a potential tool to indicate botanical and geographical origin. Besides the two main constituents of honey, which are glucose and fructose, there are about 25 other oligosaccarides (disaccarides, trisaccarides, and tetrasaccarides), which occur as relatively minor components. In Table $\mathbf{4}$, we report the range and mean levels of glucose, fructose, maltose, raffinose, and saccharose in the 70 samples analyzed. Maltose and raffinose were selected as two oligosaccharide parameters to be used in the classification of Turkish honey with the presence of maltose being characteristic of both rhododendron and honeydew honeys. Raffinose and maltose rank as second and fourth, respectively, in variable importance in the PLS-LDA of all the 51 variables (see Table 1).

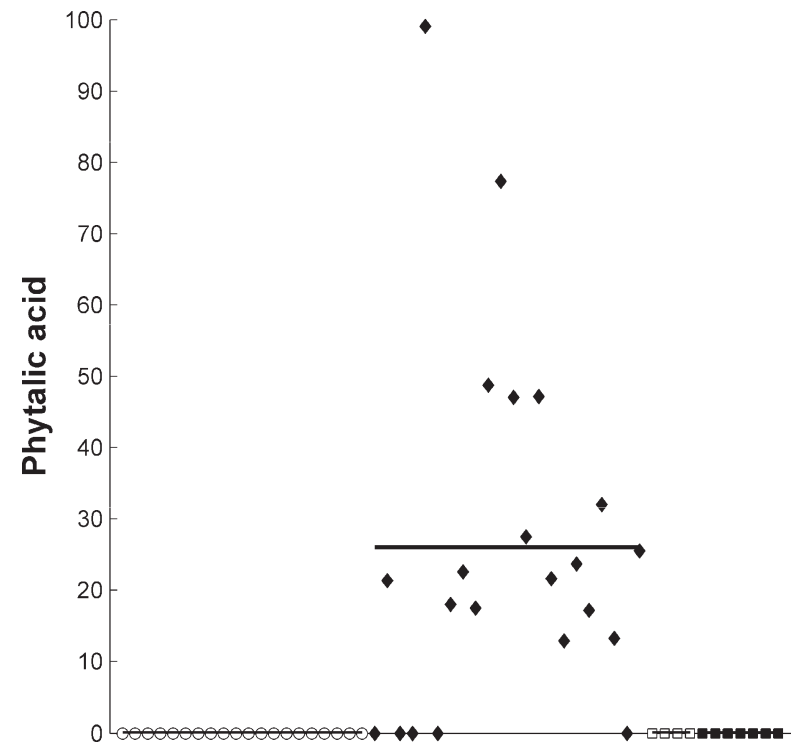

Figure 4. GC-peak area of phthalic acid in rhododendron (O), multifloral $(\diamond)$, chestnut $(\square)$, and Anzer $(\square)$ honeys.

However, there were some exceptions with one of the 20 rhododendron honeys from Ordu being found to contain $<0.6 \mathrm{~g} / 100 \mathrm{~g}$ of maltose. One Anzer and one chestnut honey sample were also found to contain 4.41 and $1.8 \mathrm{~g} / 100 \mathrm{~g}$ of maltose, respectively, again demonstrating that for almost all marker compounds there were exceptions for classification purposes. Five samples of the 24 multifloral honeys were also found to contain maltose at levels ranging from 3.3 to $5.1 \mathrm{~g} / 100 \mathrm{~g}$, although it is of course possible that there may have been contributing rhododendron pollen in these samples. Maltose was not found in either the citrus or sunflower samples, but it is difficult to draw conclusions from single samples. In Brazilian honey (10), levels of maltose ranged from 0.2 to $6.8 \mathrm{~g} / 100 \mathrm{~g}$, which are consistent with the levels we have found in Turkish honey.

Raffinose was present at relatively low levels in all 70 samples of honey, and there were some apparent differences in the levels found between different honey floral types. Raffinose ranked second among the variables contributing to discrimination, and maltose ranked fourth in this listing (Table 1). Figure 5 shows a plot of raffinose concentrations showing some degree of separation; in particular, rhododendron has a clear separation from the other honeys with multifloral and chestnut showing similar groupings. A study of 91 English honey samples for 40 oligosaccharides and a combination of hierarchical cluster analysis, PCA, and canonical discriminant analysis showed that it was possible, with some exceptions, to discriminate floral 


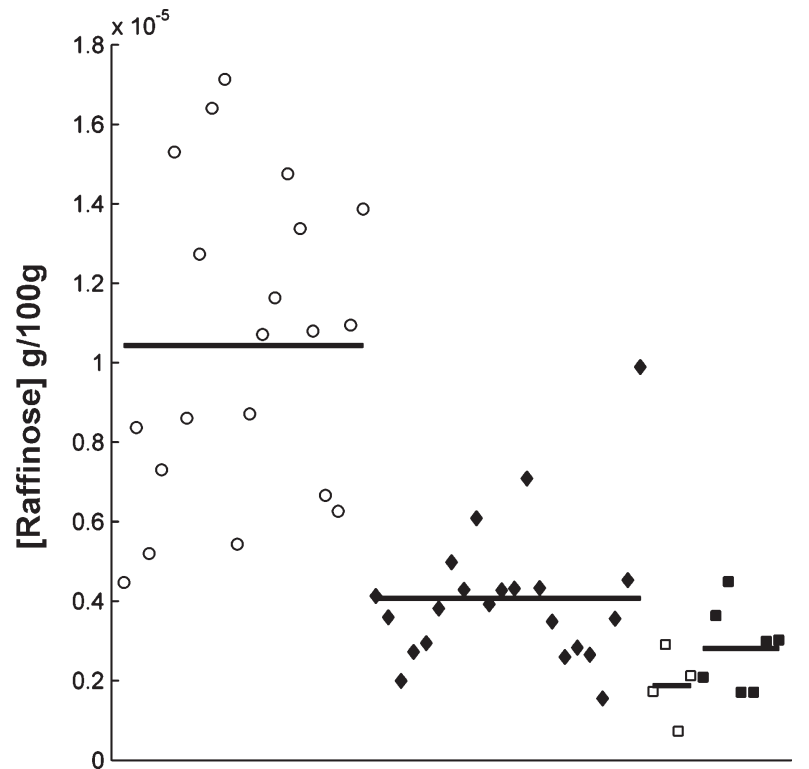

Figure 5. Concentration of raffinose in rhododendron $(\bigcirc)$, multifloral $(\diamond)$, chestnut ( $\square$ ), and Anzer ( $\square$ ) honeys.

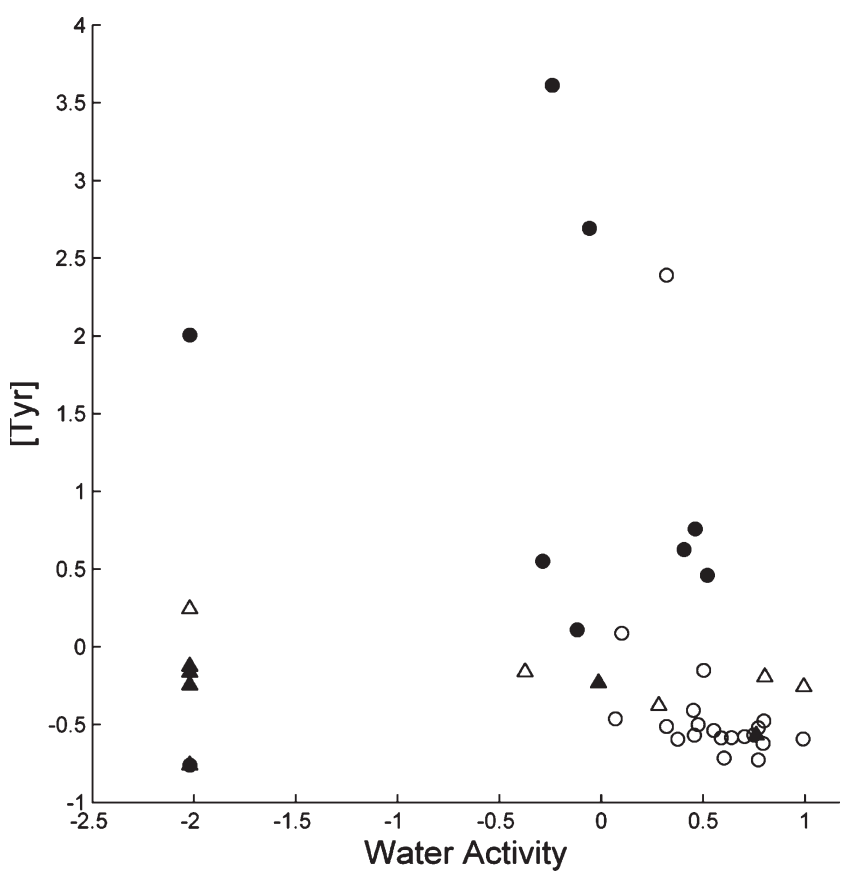

Figure 6. Analysis of water activity and tyrosine data with mean-centering showing the discrimination of rhododendron $(O)$, chestnut $(\Delta)$, honeydew $(\mathbf{\Delta})$, and Anzer (๑) Turkish honeys.

types, but unfortunately, these authors did not indicate which of the oligiosaccharides contributed most to the discrimination (11). Maltose can also be used as a geographical as well as botanical marker. In work on Brazilian honey, the contents of maltose, nigerose, turanose, and maltotriose also proved to be useful for the differentiation of honey samples from different geographical regions (10).

Most publications in the area of honey authenticity have attempted to use single data sets of parameters such as volatiles, amino acids, or carbohydrates combined with multivariate analysis to look for individual markers or combinations of parameters that could be used for discrimination. Although this approach has had some success, results have rarely shown unequivocal discrimination between samples. Surprisingly there are few papers where multiple analytes have been determined in the same samples of honey and where the parameters showing maximum discrimination in one set have been combined with those from another set. Thus, in principle it should be possible to combine one or two amino acids with selected marker volatiles and perhaps also with some oligosaccharides to achieve improved discrimination. Most researchers have attempted to focus on the development of practical tools that could be used in food control and enforcement situations, and therefore, methodology requiring databases of a range of parameters where different analytical methods are required might be seen as impractical. However, as a practical example of this approach we have used a combination of levels of water activity with levels of only the amino acid tyrosine as can be seen in the plots shown in Figure 6. Here, it is evident that rhododendron honey with the exception of a single sample can be clearly distinguished from chestnut, honeydew, and Anzer honeys. This simpler approach of basing measurements on only two parameters might offer a more practical tool than that based on the need to generate large datsets.

This article reports for the first time an exhaustive analysis of authenticated samples of Turkish honey of different geographical regions and of differing floral types. This includes new data on Anzer and rhododendron honey samples, which are unique to Turkey. We have shown that a combined data set of amino acids, volatiles, saccharides, and water activity measurements allows the floral origin of Turkish honey to be accurately predicted and thus provides a useful tool for authentication purposes. However, using variable selection techniques a smaller subset of analytes has been identified that has the capability of classifying Turkish honey according to floral type with a similar level of accuracy.

\section{ACKNOWLEDGMENT}

Sebnem Ozturkoglu and Sureyya Ozcan are acknowledged for their tremendous help.

\section{LITERATURE CITED}

(1) Louveaux, J. Atlas photographique d'analyse pollinique des miels. Paris. Serv Repress Fraud. Contr. Qualite, Min. Agric. 1970

(2) Alissandrakis, E.; Tarantilis, P. A.; Harizanis, P. C.; Polissiou, M. Comparison of the volatile composition in thyme honeys from several origins in Greece. J. Agric. Food Chem. 2007, 55, 8152-8157.

(3) Perez, R. A.; Sanchez-Brunete, C.; Calvo, R. M.; Tadeo, J. L. Analysis of volatiles from Spanish honeys by solid phase microextraction and gas chromatography-mass spectrometry. J. Agric. Food Chem. 2002, 50, 2633-2637.

(4) Verzera, A.; Campisi, S.; Zappala, M.; Bonaccorsi, I. SPME-GCMS analysis of honey volatile components for the characterization of different floral origin. Am. Lab. 2001, 18-21.

(5) Soria, A. C.; Martinez-Castro, I.; Sanz, J. Analysis of volatile composition of honey by solid phase microextraction and gas chromatography-mass spectrometry. J. Sep. Sci, 2003, 26, 793-801.

(6) Cuevas-Glory, L. F.; Pino, J. A.; Santiago, L. S.; Sauri-Duch, E. A review of volatile analytical methods for determining the botanical origin of honey. Food Chem. 2007, 103, 1032-1043.

(7) Hermosin, I.; Chicon, R. M.; Cabezudo, M. D. Free amino acid composition and botanical orgin of honey. Food Chem. 2003, 83, 263-268.

(8) Gonazalez-Paramas, A. M.; Gomez Barez, J. A.; Cordon Marcos, C.; Garcia-Villanova, R. J.; Sanchez, J. S. HPLC-fluorimetric method for analysis of amino acids in products of the hive (honey and bee pollen). Food Chem. 2006, 95, 148-156.

(9) Gilbert, J.; Shepherd, M. J.; Wallwork, M. A.; Harris, R. G. Determination of the geographical origin of honeys by multivariate analysis of gas chromatographic data on their free amino acid content. J. Apicultural Res. 1981, 20, 125-135.

(10) Costa Leite, Da J.M.; Trugo, L. C.; Costa, L. S. M.; Quinterio, L. M. C.; Barth, O. M.; Dutra, V. M. L.; Maria, C. A. B. Determination of 
oligosaccharides in Brazilian honeys of different botanical origins. Food Chem. 2000, 70, 93-98.

(11) Goodall, I.; Dennis, M. J.; Parker, I.; Sharman, M. Contribution of high-performance liquid chromatographic analysis of carbohydrates to authenticity testing of honey. J. Chromatogr., A 1995, 706, 353-359.

(12) Lachman, J.; Kolihova, D.; Miholova, D.; Kosata, J.; Totera, D.; Kult, K. Analysis of minority honey components; Possible use for the evaluation of honey quality. Food Chem. 2007, 101, 973-979.

(13) Ferreres, F.; Tomas-Baberan, F. A.; Gil, M..; Tomas-Lorente, F. An HPLC technique for flavanoid analyis in honey. J. Sci. Food Agric. 1991, 56, 49-56.

(14) Wilkins, L. A.; Lu, Y.; Tan, S. T. Extractives from New Zealand honeys. 5. Aliphatic dicarboxylic acids in New Zealand Rewarewa honey. J. Agric. Food Chem. 1995, 43, 3021-3025.

(15) Jorg, E.; Sontag, G. Determination of phennolic acids in honey by HPLC using coulometric duel electrode detection. Dtsch. Lebensm.Rundsch. 1992, 88, 179-183.

(16) Popek, S. A procedure to identify a honey type. Food Chem. 2002, 79, 401-406.

(17) Corbella, E.; Cozzolino, D. Classification of the floral origin of Uruguayan honeys by chemical and physical characteristics combined with chemometrics. LWT Food Sci. Technol. 2006, 39, 534539.

(18) Spano, N.; Ciulu, M.; Floris, I.; Panzanelli, A.; Pilo, M. I.; Piu, P. C.; Scanu, R.; Sanna, G. Chemical characterization of a traditional honey-based Sardinian product: Abbamele. Food Chem. 2008, 108, $81-85$.

(19) Anklam, E. A review of the analytical methods to determine the geographical and botanical origin of honey. Food Chem. 1998, 63, 549-562.

(20) Silici, S.; Gokceoglu, M. Pollen analysis of honeys from Mediterranean regions of Anatolia. Grana 2007, 46, 57-64.

(21) Pendleton, M. Description of melissoplaynological methods involving centrifugation should include data for calculating Relative Centrifugal Force (RCF) or should express data in units of RCF or gravities (g). Grana 2006, 45, 71-72.

(22) Ozcan, S.; Senyuva, H. J. Improved and simplified liquid chromatography/atmospheric pressure chemical ionization mass spectrometry method for the analysis of underivatized free amino acids in various foods. J. Chromatogr., A 2006, 1135, 179-185.

(23) Chirife, J.; Zamora, M. C.; Motto, A. The correlation between water activity and \% moisture in honey: Fundamental aspects and application to Argentine honeys. J. Food Eng. 2006, 72, 287-292.

(24) Cometto, P. M.; Faye, P. F.; Paola, R. D.; Naranjo, R. D. D. P.; Rubio, M. A.; Aldao, M. A. J. Comparison of free amino acids profile in honey from three Argentinian regions. J. Agric. Food Chem. 2003, 51, 5079-5087.

(25) Gonzalez-Paramas, A. M.; Garcia-Villanova, R. J.; Alfonso Gomez Barez, J.; Sanchez, J. S.; Albajar, R. A. Botanical origin of monovarietal dark honeys (from heather, holm oak, pyrenean oak and sweet chestnut) based on their chromatic characters and amino acid profiles. Eur. Food Res. Technol. 2007, 226, 87-92.

(26) Radovic, B. S.; Careri, M.; Mangia, A.; Musci, M.; Gerboles, M; Anklam, E. Contribution of dynamic headspace GC-MS analysis of aroma compounds to authenticity testing of honey. Food Chem. 2002, 72, 511-520.

(27) Alissandrakis, E.; Tarantalis, P. A.; Harizanis, P. C.; Polissious, M. Aroma investigation of unifloral Greek citrus honey using solidphase microextraction coupled to gas chromatographic-mass spectrometric analysis. Food Chem. 2007, 100, 396-404.

(28) Glory-Cuevas, L. F.; Pino, J. A.; Santiago, L. S.; Sauri-Duch, E. A review of volatile analytical methods for determining the botanical origin of honey. Food Chem. 2007, 103, 1032-1043.

(29) Dortterl, s.; Burkhardt, D.; Weibbecker, B.; Jurgens, A.; Schutz, S.; Mosandl, A. Linalool and lilac aldehyde/alchol in flower scents. Electrophysiological detection of lilac aldehyde stereoisomers by a moth. J. Chromatogr., A 2006, 1113, 231-238.

(30) Tasdemir, D.; Demirci, B.; Demirici, F.; Donmez, A. A.; Husnu Can Baser, K.; Ruedi, P. Analysis of the volatile components if five Turkish rhododendron species by headspace solid-phase microextraction and GC-MS (HS-SPME-GC-MS). Z. Naturforsch. 2003, $58 c, 797-803$

Received for Review January 5, 2009. Revised manuscript received March 12, 2009. Accepted March 18, 2009. We gratefully acknowledge the TUBITAK-ATAL Directorate for financial support. 